\title{
Modélisation de la diversité des systèmes de culture, de l'observation à la détection : cas de l'oasis de Nefta (Djérid, Tunisie)
}

\author{
Mireille Fargette ${ }^{1, *}$, Maud Loireau ${ }^{1}$, Najet Raouani ${ }^{2}$, Moufida Kadri Zara ${ }^{3}$, Héloïse Simon ${ }^{1}$, \\ Thérèse Libourel ${ }^{4}$, Mongi Sghaier ${ }^{5}$ et Didier Leibovici ${ }^{6}$ \\ ${ }^{1}$ UMR 228, Espace-Dev, IRD, Maison de la Télédétection, 500 rue Jean-François Breton, 34093 Montpellier Cedex 5, France \\ 2 Institut supérieur agronomique de Chott-Mariem, UR13, AGR04, université de Sousse, Sousse, Tunisie \\ ${ }^{3}$ Commissariat régional du développement agricole, Tozeur, Tunisie \\ ${ }^{4}$ UMR 228, Espace-Dev, université Montpellier, Maison de la Télédétection, 500 rue Jean-François Breton, 34093 Montpellier Cedex 5, \\ France \\ ${ }^{5}$ Institut des régions arides, Médenine, Tunisie \\ ${ }^{6}$ Nottingham Geospatial Institute, University of Nottingham, Nottingham, UK
}

\begin{abstract}
Résumé - Ce travail propose une approche par modélisation interdisciplinaire pour décrire et détecter la diversité des systèmes de culture dans les oasis du Maghreb. La méthode conjugue des concepts issus de la sémiotique et de la systémique. Elle s'appuie sur des méthodes de statistique multivariée utilisant des jeux indépendants de données d'observation. Une typologie de systèmes de culture est d'abord construite par analyse des correspondances multiples, à partir de données agronomiques collectées sur place par observation directe ou enquêtes de terrain. Il est ensuite recherché, par analyse factorielle discriminante, la fonction d'association qui relie chaque type de système de culture à la physionomie des parcelles de culture, celle-ci ayant été renseignée par des données géométriques et radiométriques détachées du terrain. La qualité estimée de l'association confère une capacité indicatrice aux données physionomiques utilisées, que cela soit pour détecter le «comment», le «où», ou le «résultat» du système de culture. Ce travail exploratoire est mené sur l'oasis de Nefta dans le Djérid tunisien. Le potentiel en généricité de la démarche construite est discuté dans sa contribution aux observatoires sociétés-milieux au service des sociétés oasiennes et des gestionnaires de territoires en zones arides.
\end{abstract}

Mots clés : agrosystème / système de culture / physionomie / indicateur / observatoire

\begin{abstract}
Modelling the cropping system diversity, from observation to detection: the case of the Nefta Oasis (Djerid, Tunisia). The purpose of this research is to establish a methodology to describe and detect the cropping system diversity in the Maghreb oases from an interdisciplinary approach. The method calls for concepts originating from semiotics and systemics domains together with multivariate statistics on independent sets of observed data. First, different types of cropping systems have been extracted from multiple correspondence analysis of surveyed agronomic variables collected in situ (direct observations and measures and/or obtained from interviews). Then, a discriminant factorial analysis was used to assess the quality of the predictive associations between these types and datasets covering a range of characteristics from geometric and radiometric indicators accounting for culture plots "physiognomy", the data collection of which did not require here any field survey. Whether to detect the "how", the "where" or the "what" of the cropping system, the method appears to reach an encouraging discriminating capacity. This pilot study was carried out in the Nefta Oasis, Djerid, Tunisia. The generic aspect of the approach and its potential are discussed, as contribution to population-environment observatories for Oasis society and territorial managers in arid zones.
\end{abstract}

Keywords: agro-ecosystem / cropping system / physiognomy / indicator / observatory

\footnotetext{
* Auteur de correspondance : Mireille.Fargette@ird.fr
} 


\section{Introduction}

Pour mieux comprendre et suivre les évolutions spatiotemporelles des phénomènes socio-environnementaux affectés par les changements globaux et in fine fournir une information utile à la gestion des territoires, il est crucial de disposer de méthodes et outils adaptés à la fois en matière de rapidité et facilité d'acquisition de données et de potentiel de déploiement sur de larges espaces.

Parmi les systèmes interactifs entre les hommes et leurs milieux, les agrosystèmes et les systèmes de culture (Sébillotte, 1990) qui leur sont associés, évoluent rapidement. Or, ces derniers jouent un rôle important pour la sécurité alimentaire, la dégradation des sols et la pauvreté. Aussi méritent-ils une attention particulière.

Le travail s'intéresse à l'agrosystème oasien, emblématique des zones arides du Maghreb. Dans un contexte de contraintes naturelles sévères, les oasis ont montré leur adaptabilité au cours du temps, ainsi que leur rôle fondamental dans le développement des zones arides (Kassah, 2009).

Pour caractériser et suivre, dans le temps et dans l'espace, les évolutions des agrosystèmes, un enjeu méthodologique majeur consiste à détecter de manière rapide, facile et fiable, l'expression de la diversité des agrosystèmes. Nous avons donc recherché une méthode économe en données collectées sur place, pouvant couvrir de grands espaces et reproductible.

Le postulat de base est qu'il existe un lien entre l'agrosystème mis en œuvre sur un espace et la physionomie que celui-ci revêt. Deffontaines (2004) mentionne les liens entre les objets du paysage agricole et les processus en jeu. Que cela soit dans le domaine de la géographie (Brunet, 1974; Claval, 1974) comme dans celui de l'agronomie (Michelin, 2008), ces liens de type système-physionomie ou système-empreinte sont à rapprocher de ce que le champ de la sémiotique étudie dans la théorie générale des signes sous toutes leurs formes et toutes leurs manifestations. En conséquence et toujours selon ce postulat, l'évolution des agrosystèmes peut être suivie par l'évolution de la physionomie des espaces concernés.

En s'appuyant sur ce postulat, l'objectif de ce travail est de construire une démarche générique pour distinguer la diversité que déploie dans l'espace un agrosystème, en l'occurrence l'agrosystème oasien, via ses systèmes de culture. La méthode exploratoire est décrite et appliquée sur la zone d'étude, l'oasis de Nefta dans le Djérid tunisien. Les premiers résultats qui en découlent sont présentés et leur place et contribution discutées dans le dispositif général d'observatoire des territoires oasiens.

\section{Matériel et méthode}

\subsection{Du système de culture à la parcelle culturale observée}

Un système de culture (Sébillotte, 1990), noté ici SC, est caractérisé par une combinaison de pratiques culturales (irrigation, amendement, association de culture...) que l'agriculteur déploie sur des surfaces selon des logiques de positionnement géographique (distance aux forages ou à la route, topographie locale...). Le SC détermine un type d'état de surface (couvert végétal, surface du sol) et la présence d'objets (Barthes, 1953) et traces qu'il produit (puits, clôtures, brisevent, canaux...).
Une parcelle culturale correspond à une unité de surface continue sur laquelle est mené un unique SC. En conséquence, la granularité du travail (échantillonnage, collecte de données, analyse) est celle de la parcelle culturale et non celle de la parcelle cadastrale liée à son statut foncier. C'est ainsi qu'est entendu «parcelle» dans toute la suite.

Outre le SC qui y est mené, la parcelle présente des caractéristiques environnementales déterminantes pour le SC ; les résultats obtenus de l'interaction agricole s'y expriment aussi. Cette conception du SC (comment, où, résultat) guide la structuration des données agronomiques collectées ( $c f$. encadré 2, Fig. 1).

La parcelle varie dans sa taille, sa forme (plus ou moins complexe) et sa localisation, ainsi que dans les caractéristiques d'ordre bio-physico-chimiques qu'elle donne à voir à tous types de capteurs (appareil photo, satellite...) ou observation visuelle. Que ces données relèvent du contenant ou du contenu, ou encore de la position géographique (relative ou absolue), elles sont toutes confrontées à la logique systémique du SC et sont candidates à témoigner (de près ou de loin selon le capteur) et à différencier des types de SC.

\subsection{Site d'étude}

L'oasis de Nefta est située dans le gouvernorat de Tozeur, dans le Sud-Ouest tunisien (Fig. 1a).

Oasis séculaire du Djérid tunisien, elle a connu de grandes modifications agro-environnementales au cours des dernières décennies. Des politiques publiques ont soutenu le secteur phœnicicole et l'exportation de datte de variété Deglet Nour (Conforti et Tonneau, 1999). De plus, des tarissements de sources d'eau naturelles (Battesti, 2005) sont survenus, ainsi que des «fatigues» du palmier d'ordre physiologique ou pathologique (Namsi et al., 2007; Triki et al., 2003). Depuis une décennie, les structures de développement se mobilisent pour réhabiliter l'agriculture oasienne en luttant contre le morcellement des champs et la déprise, en encourageant la vente des parcelles abandonnées malgré les liens affectifs entre la terre et ses propriétaires, en revalorisant, voire en réapprenant aux jeunes, la culture à étages (AGDOR, 2015).

La diversité des SC de l'oasis de Nefta est compatible avec l'objectif de l'étude et fait de cette oasis la zone-test de cette recherche. Elle est immédiatement perceptible en circulant dans l'oasis originelle et dans les palmeraies d'extension à sa périphérie; tous les intermédiaires sont possibles allant de parcelles très bio-diverses à la monoculture de palmier (souvent observée dans les extensions), ou encore de parcelles visiblement très entretenues aux parcelles laissées à l'abandon.

Le recueil de données sur le terrain a eu lieu de mars à juillet 2012 .

\subsection{Constitution des échantillons de parcelles}

Nous ne disposions en début d'étude d'aucune mesure de la diversité des SC faute de recensement agricole récent et détaillé, ni d'aucune information sur leur organisation spatiale agraire. Pour capturer cette réalité, nous avons constitué un échantillon de parcelles de distribution uniforme sur une surface de 1075 ha correspondant à l'ensemble de l'oasis originelle et aux extensions postérieures à 2000 . Une grille de maille carrée de $500 \mathrm{~m}$ de côté a été virtuellement apposée sur 


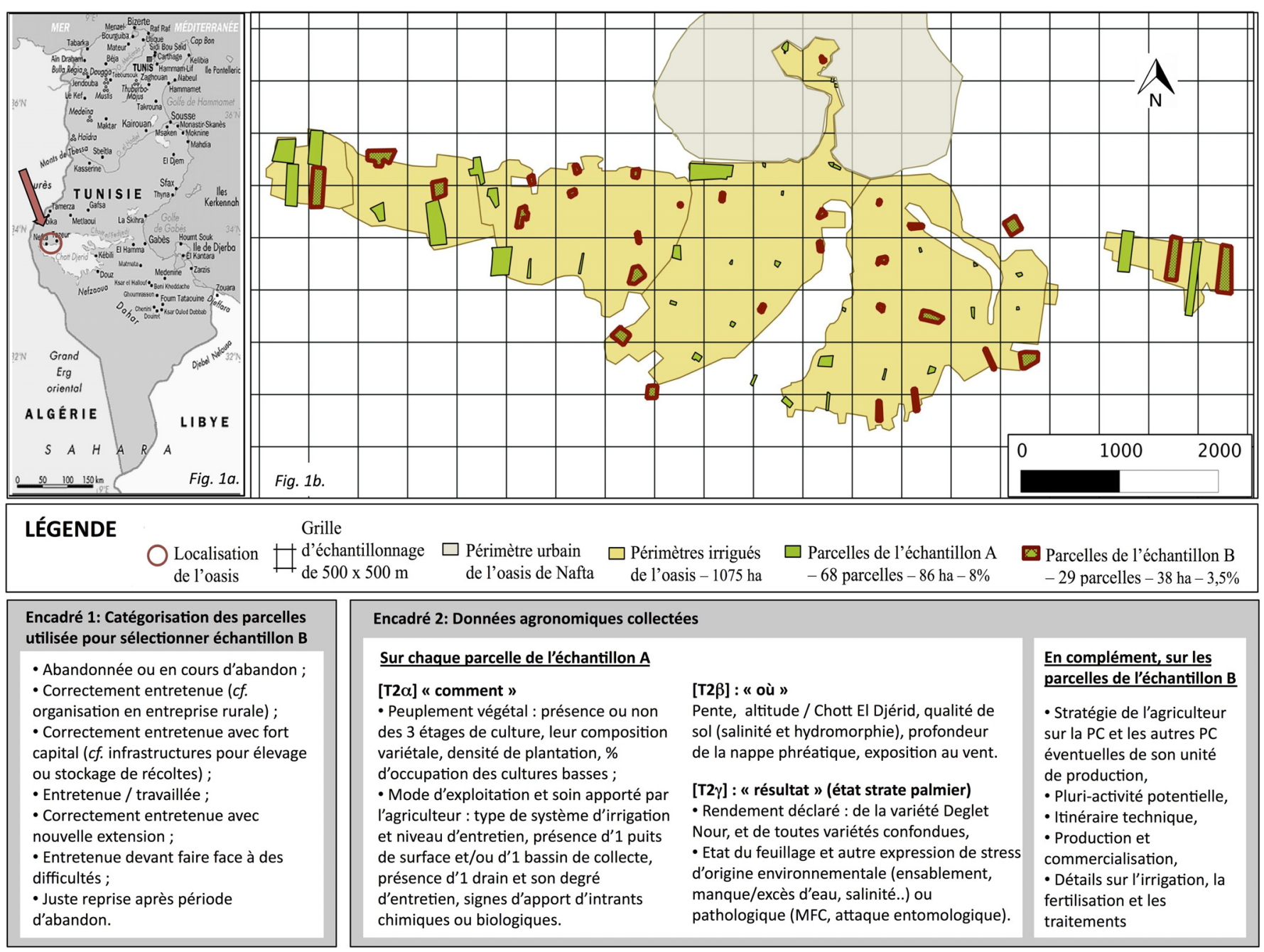

Fig. 1. Localisation de l'oasis de Nefta dans le sud tunisien (a); localisation des parcelles des échantillons A et B (b); description des variables agronomiques.

Fig. 1. Nefta Oasis location in Southern Tunisia (a); location of Sample A and Sample B plots (b); agronomic variables description.

la surface. Dans chaque maille de 25 ha, une parcelle a été retenue avec pour règle que ses coordonnées GPS soient les plus proches de celles du barycentre de la maille. Il a résulté la sélection de 68 parcelles uniformément réparties sur la zone concernée, aussi bien en son cœur qu'en bordure, y compris celle avec la ville (Fig. 1b); leurs surfaces cumulées correspondent à une superficie totale de 86 ha, soit un peu plus de $8 \%$ de la surface échantillonnée.

Pour les besoins de la méthode, les parcelles sont organisées en deux échantillons :

- l'échantillon A comprend les 68 parcelles;

- l'échantillon $\mathrm{B}$ correspond à un sous-ensemble de A. Il comprend 29 parcelles et représente la diversité perçue et la potentielle diversité technico-économique catégorisée en sept états (adaptés des travaux de Battesti, 2005; $c f$. encadré 1, Fig. 1). Il n'a pas été possible de rencontrer plus d'agriculteurs ou suffisamment longtemps, pour augmenter la taille de $\mathrm{B}$. Même réduite, celle-ci n'interdit pas de procéder à une Analyse des correspondances multiples (ACM) et une Analyse factorielle discriminante (AFD) sur le jeu de données, $c f$. la Section 3.2.

\subsection{Données relatives aux parcelles culturales}

Trois corpus de données sont obtenus pour décrire les 68 parcelles :

- données agronomiques: $[\mathrm{T}]$;

- données géométriques: [G];

- données radiométriques: [R].

\subsubsection{Données agronomiques [T]}

Il s'agit de données recueillies directement et systématiquement pour chaque parcelle de l'échantillon A, par observation sur le terrain. Deux groupes de données sont distingués :

- [T1]: une information d'ordre agronomique ( $c f$. encadré 2, Fig. 1) scindée en trois catégories $(\alpha, \beta, \gamma)$, relevant d'objets, traces et conditions des SC opérant sur la parcelle; elle est observée dans la parcelle et notée sur une fiche préalablement établie. Les types d'observation et la notation de leurs modalités ont été définis préalablement avec le concours des techniciens du Centre régional de recherche en culture oasienne (CRRAO) et des agriculteurs de Nefta. 
Des données agronomiques complémentaires ont été recueillies sur les parcelles de l'échantillon B lors d'entretiens semi-directifs auprès des agriculteurs. Elles permettent de confirmer l'information contenue en [T1], de la réajuster si nécessaire et de la compléter. L'ensemble des données observées et récoltées de l'échantillon B constitue le corpus [T'1];

- [T2]: un jeu de photos accompagnant l'information [T1].

\subsubsection{Données géométriques [G]}

Les caractéristiques géométriques retenues concernent la complexité de forme (indices classiques), la taille (surface) et le positionnement géographique (plus petites distances au centre urbain de Nefta et au Chott El Djérid, une zone endoréique asséchée). Elles sont calculées sous SIG à partir des coordonnées précises du pourtour de chaque parcelle; ce géo-référencement est obtenu par arpentage le long du périmètre et enregistrement de la trace à l'aide d'un GPS.

\subsubsection{Données radiométriques [R]}

Les données radiométriques correspondent au contenu numérique d'une image satellite SPOT 5 du mois de juin 2011, donc antérieure d'un an seulement à la campagne de terrain et correspondant à la même saison. Le géo-référencement précis du pourtour des 68 parcelles rend possible le calcul de données radiométriques rapportées à la parcelle. Pour chaque parcelle et chaque type de données radiométriques, la moyenne des valeurs portées par les pixels contenus dans la parcelle est calculée. On obtient ainsi :

- les valeurs moyennes des bandes spectrales [BS];

- les valeurs moyennes d'indices radiométriques classiques porteurs d'information sur la végétation ou le sol [IR].

\subsection{Démarche et analyse multivariée}

La démarche comporte deux parties, enchaînant cinq étapes dans le raisonnement. La première partie a pour objectif de construire une typologie à partir de l'information agronomique ; la seconde a pour objectif de construire une méthode générique (non inféodée au type de données) de détection des types, à partir d'un jeu de données indépendant de celui ayant servi à construire la typologie et pensé pour s'obtenir sans l'obligation du recours à une campagne de terrain. L'indépendance d'acquisition des données utilisées pour la construction de la typologie des SC d'une part, pour la mesure de la qualité de leur détection d'autre part, garantit la validité de cette mesure.

\subsubsection{Partie 1 : construction de la typologie}

Étape 1: sur l'échantillon B de 29 parcelles et à partir du corpus de données [T'1] l'analyse multivariée (analyse des correspondances multiples $[\mathrm{ACM}]+$ classification ascendante hiérarchique $[\mathrm{CAH}])$ permet une typologie.

\subsubsection{Partie 2: construction et application de la méthode de détection des types}

Étape 2 : sur ce même échantillon $B$, les types obtenus sont confrontés par analyse factorielle discriminante (AFD) aux données des corpus $[\mathrm{G}]$ et $[\mathrm{R}]$ : apprentissage de la fonction d'association.

Étape 3: la fonction d'association est appliquée sur l'ensemble des 68 parcelles de l'échantillon A. L'inférence produite attribue un type à chaque parcelle: prédiction.

Étape 4 : pour les 29 parcelles d'apprentissage, on constate directement les bons/mauvais classements. Pour les 39 parcelles restantes, la non-conformité (i.e., l'incohérence de la proposition inférée par rapport aux observations enregistrées en [T1] et [T2]) est notée. Le score de non-conformité est enregistré (nombre de parcelles mal typées / total) : estimation de la qualité de la fonction d'association.

Étape 5: le poids des variables géométriques et radiométriques dans la prédiction des types est estimé en réitérant les étapes 2, 3 et 4 pour différentes sélections de variables. Il ne s'agit pas de faire une analyse complète de leur redondance mais plutôt de caractériser conceptuellement l'apport du géométrique, des indices radiométriques et des valeurs des bandes spectrales, chacun dans leur ensemble.

\section{Résultats}

Le déploiement de la démarche n'est détaillé que pour les données agronomiques de la catégorie $\alpha$ du corpus [T1], caractéristique du SC (comment). La même démarche étant appliquée aux catégories $\beta$ (où) et $\gamma$ (résultat) du corpus [T1], seuls les typologies et scores de qualité de la fonction d'association sont fournis en fin de Sections 3.1 et 3.2.

\subsection{Typologie des systèmes de culture}

L'information agronomique (quantitative ou qualitative) très fournie du [T'1 $\alpha]$ est combinée en 14 variables descriptives des SC. Ces variables se déclinent en 31 modalités qualitatives:

- l'ACM analyse les «corrélations» entre les 14 variables descriptives et propose l'organisation de leurs modalités sur le plan factoriel F1-F2 (Fig. 2) qui cumule $82 \%$ de l'information;

- sur la base de la recomposition de l'information selon ce nouveau repère, la $\mathrm{CAH}$ organise les 29 parcelles en quatre groupes, chacun illustrant un type de SC;

- la considération des combinaisons de modalités de variables présentées par les parcelles d'un même groupe permet de dégager «l'association-type» de variables (ou de modalités de variables) préférentiellement partagées par ces parcelles, caractéristique d'un SC. Toutes les variables ne seront donc pas systématiquement mentionnées dans chacun des profils-types décrits ( $c f$. description textuelle et illustration photographique, Fig. 3);

- nous constatons que les limites entre regroupements de variables par type ne sont pas très marquées. Ceci ne remet pas en cause la méthodologie en soi (voir aussi la Section 3.2).

Pour illustrer de manière synthétique les différences entre les types de SC obtenus, les variables sont distribuées dans quatre thèmes ( $c f$. encadré, Fig. 4) selon des hypothèses métier (agronomie) sur leur contribution potentielle dans chacun d'eux. Chaque type de SC peut être représenté de façon 


\section{Axes $\mathrm{F} 1$ et $\mathrm{F} 2: 82,43 \%$}

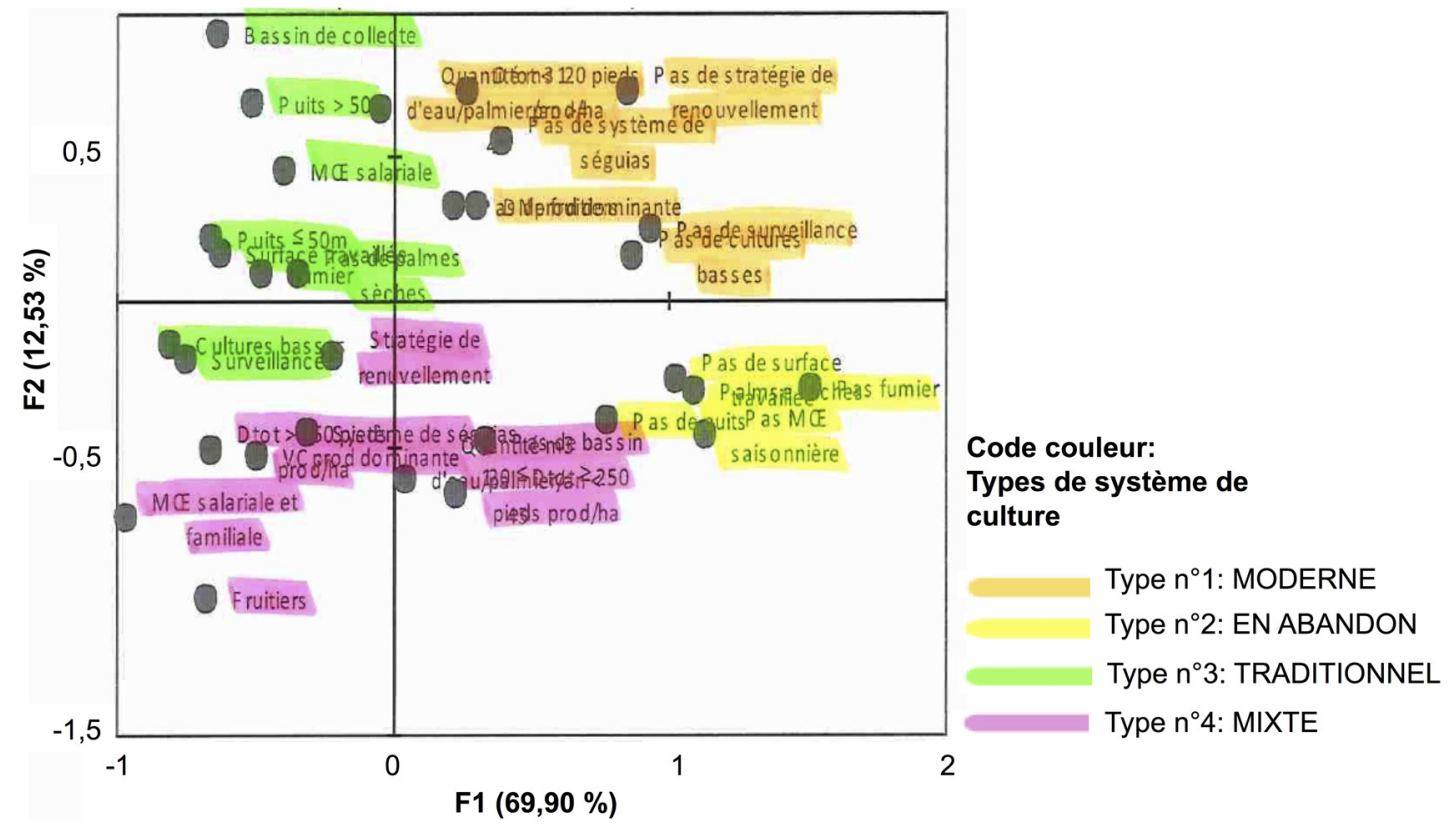

Fig. 2. Représentation des 31 modalités des 14 variables sur le plan factoriel F1-F2 résultant de l'analyse des correspondances multiples, effectuée sur l'échantillon B.

Fig. 2. Scatter-plot of the variable categories on the first two principal dimensions of the Multiple Correspondence Analysis performed on Sample B.

synthétique (Fig. 4) en le caractérisant par un score (de 0 à 7) pour chacun des thèmes (axes du diagramme). Cette représentation permet d'apprécier rapidement les particularités de chaque SC et de les comparer.

Les deux autres typologies produites à partir des variables de chacune des deux autres catégories du corpus [T1] correspondent à :

- parcelle en milieu (i) favorable, (ii) difficile car salin, (iii) difficile car hydromorphe (catégorie $\beta$ );

- état de la strate palmier : bon, moyen, mauvais (catégorie $\gamma$ ).

\subsection{Qualité de la fonction d'association}

En référence à l'étape 4 de la démarche, nous tenons compte dans un premier temps des attributions possiblement non conformes au prédicat (non-conformité stricte) et aboutissons à un score de non-conformité de 6/68 dans l'échantillon A, 1/29 dans l'échantillon d'apprentissage B, et 5/39 dans l'échantillontest (le reste de l'échantillon $=\mathrm{A}-\mathrm{B}$ ). Le faible taux de mal classé dans l'échantillon B permet d'avoir une certaine confiance à la fois dans la cohérence de la typologie et dans le potentiel d'indication que l'on recherche dans les jeux de données [G] et [R]. Ceci est un premier résultat.

Dans un second temps, nous retenons comme non conformes des attributions conformes mais qui, selon l'information disponible dans les jeux de données [T1] et [T2], peuvent aussi aller à un autre type (non-conformité élargie). Dans ce cas, les valeurs de non-conformité augmentent pour atteindre 13/68 dans l'échantillon A, 4/29 dans l'échantillon B et 9/39 dans [A - B]. En augmentant l'exigence sur la détection, la fonction d'association reste cependant indéniablement efficace.
La taille réduite de l'échantillon B aurait pu limiter les chances de succès de la méthode. En effet, son manque possible de représentativité aurait pu tout à la fois remettre en cause la typologie dans sa pertinence (incomplète voire erronée) ou sa solidité (qualité de résolution des types). Une AFD fondée sur un échantillon qui aurait manqué de représentativité aurait eu peu de capacité de détection, sa fonction d'association ne retrouvant pas la logique de l'échantillon d'apprentissage dans l'échantillon-test. Au contraire, la méthode montre des taux de succès de classification tout à fait satisfaisants.

Néanmoins, les groupements de variables à l'origine de la typologie étaient peu marqués, i.e., associations «floues » non forcément exclusives. Nous pensons à une caractéristique de nature intrinsèque des SC (ou des mesures utilisées pour les décrire) ayant tendance à exprimer un continuum par rapport au contexte biophysique et/ou social de l'oasis. Ces associations «floues» qui ne peuvent pas être dues à une taille d'échantillon expliquent les augmentations des taux de succès entre conformité stricte et élargie.

Quoi qu'il en soit, étant donnés les taux de succès de détection (succès global sur l'échantillon $\mathrm{A}$ : $91,2 \%$ et $81 \%$ selon les deux critères de conformités utilisés ; $87,2 \%$ et $77 \%$ sur l'échantillon-test [A - B]), la méthodologie en soi n'est pas remise en cause et le potentiel de détection de la fonction d'association semble prometteur.

En référence à l'étape 5 , les résultats montrent aussi (Tab. 1) que si les bandes spectrales [BS], comme les indices radiométriques [IR], semblent avoir un meilleur potentiel de détection des SC $(60 \%)$ que les données géographiques [G] $(53 \%)$, la combinaison des trois augmente ce potentiel qui devient substantiel (69\%). 


\begin{tabular}{|c|c|c|c|}
\hline $\begin{array}{l}\text { Type } \mathrm{n}^{\circ} 1 \\
\text { MODERNE } \\
\text { Le plus } \\
\text { représenté : } \\
\text { 40\% des PC de } \\
\text { l'échantillon B } \\
\text { Remarques complémentaires } \\
\text { - Apparition assez tardive dans les oasis } \\
\text { tunisiennes, avec notamment les subventions de } \\
\text { l'état ou l'investissement de collectifs privés } \\
\text { autorisant I'installation de nouvelles } \\
\text { infrastructures hydrauliques; } \\
\text { - Répond à } 1 \text { stratégie commerciale de revenu } \\
\text { rapide par l'exportation de datte vers I'Europe. }\end{array}$ & $\begin{array}{l}\text { Description synthétique } \\
\text { - Fort investissement dans } \\
\text { infrastructures hydrauliques } \\
\text { (puits + bassin); } \\
\text { - Quantité d'eau d'irrigation } \\
\text { conséquente : }>45 \mathrm{~m}^{3} / \mathrm{pied} / \mathrm{an} ; \\
\text { - Densité faible de plantation en } \\
\text { palmier dattier : }<120 \text { pieds/ha; } \\
\text { - Variété Deglet Nour dominante ; } \\
\text { - Main d'œuvre salariale pour les } \\
\text { travaux saisonniers ; } \\
\text { - Effforts et investissements } \\
\text { dirigés vers la production de } \\
\text { datte (ni cultures basses ni arbres } \\
\text { fruitiers); } \\
\text { - Pas de renouvellement } \\
\text { progressif des palmiers. }\end{array}$ & $\begin{array}{l}\text { Remarques complémentaires } \\
\text { Les PC en abandon ou quasi-abandon } \\
\text { n'ont jamais de puits, ni de bassin de } \\
\text { stockage d'eau. }\end{array}$ & $\begin{array}{l}\text { Description synthétique } \\
\text { Abandon majeur, total ou partiel ; } \\
\text { Aucune main d'œuvre agricole } \\
\text { mobilisée : le sol n'est pas travaillé, les } \\
\text { cultures basses sont absentes, pas } \\
\text { d'apport de fumier ; les palmes sèches } \\
\text { au sol ne sont pas éliminées. }\end{array}$ \\
\hline $\begin{array}{l}\text { Type } \mathrm{n}^{\circ} \mathbf{3} \\
\text { TRADITIONNEL } \\
\text { Autant que les PC } \\
\text { en abandon : } \\
20 \% \text { des } \mathrm{PC} \text { de } \\
\text { l'échantillon B } \\
\text { Remarques complémentaires } \\
\text { - Grand savoir-faire oasien hérité de l'histoire } \\
\text { pour gérer le fragile équilibre entre ressources } \\
\text { rares (terre, eau) et cultures intensives; } \\
\text { - La plus intensive en travail (main d'œuvre } \\
\text { saisonnière salariale et familiale); } \\
\text { - Témoins d'entretien : sol nettoyé (gestion du } \\
\text { parasitisme et facilitation des travaux), palmes } \\
\text { sèches utilisées (clôtures, abri). }\end{array}$ & $\begin{array}{l}\text { Description synthétique } \\
\text { - } 3 \text { étages de cultures : palmier } \\
\text { dattier (mono- ou pluri-variétal), } \\
\text { arbres fruitiers (mono- ou pluri- } \\
\text { spécifiques), cultures basses } \\
\text { annuelles (maraîchères et/ou } \\
\text { fourragères); } \\
\text { - Mélange de variétés de palmiers } \\
\text { majoritairement communes; } \\
\text { - Densité élevée : de } 120 \text { à } 350 \\
\text { pieds/ha; } \\
\text { - Sol amendé en fumier et } \\
\text { travaillé ; } \\
\text { - Quantités d'eau d'irrigation } \\
\text { moindres : <45 } \mathrm{m}^{3} / \text { pied/an; } \\
\text { - Présence facultative de puits et } \\
\text { bassins de stockage d'eau; } \\
\text { - Acheminement de l'eau via des } \\
\text { séguias creusées dans la terre, } \\
\text { parfois renforcées de ciment; } \\
\text { - Entretien conséquent. }\end{array}$ & $\begin{array}{l}\text { Type } n^{\circ} 4 \\
\text { MIXTE } \\
20 \% \text { des PC de } \\
\text { l'échantillon B }\end{array}$ & $\begin{array}{l}\text { Description synthétique } \\
\text { - Toujours un travail fourni : apport de } \\
\text { fumier, travail du sol, entretien des } \\
\text { palmiers, même si le renouvellement } \\
\text { progressif des palmiers est faible ; } \\
\text { - Toujours un investissement en } \\
\text { structures d'accès à l'eau : puits, bassin } \\
\text { de stockage d'eau; } \\
\text { - Surtout des mélanges de variétés } \\
\text { communes avec ou sans Deglet Nour ; } \\
\text { - Densité de plantation plus faible } \\
\text { qu'en système traditionnel ; } \\
\text { - Autres critères: tous types de } \\
\text { situations intermédiaires. }\end{array}$ \\
\hline
\end{tabular}

Fig. 3. Description et illustration photographique des quatre types de système de culture.

Fig. 3. Description and photographic representations of each cropping system type.

La fonction d'association entre type de SC et physionomie telle que résumée par des variables géométriques et radiométriques est suffisamment forte pour admettre un pouvoir indicateur (capacité à détecter la diversité des SC) aux jeux de données [G], [BS] et [IR]. Même s'il faut garder à l'esprit que l'échantillon d'apprentissage est de taille réduite et que le test de non-conformité a tendance à optimiser le taux de succès, on peut reconnaître le potentiel de la méthode dès cette phase exploratoire du travail.

L'analyse des catégories $\beta$ et $\gamma$ du corpus [T1], réalisée selon la même démarche, présente respectivement un taux de succès de $90 \%$ et $75 \%$ (ou respectivement $84 \%$ et $70 \%$, selon le critère plus sévère de conformité). Ceci confirme le potentiel qu'ont des données appartenant aux registres radiométrique et géométrique à rendre compte d'une information agronomique, que celle-ci soit de catégorie $\alpha$ (comment), $\beta$ (où) ou $\gamma$ (résultat).

\section{Discussion}

Nous avons exploité la relation qui existe entre type de système de culture et physionomie de parcelle, en tant qu'espace où s'exerce le système et où il laisse son empreinte.
Nous avons opéré un «changement de façon d'observer» une oasis, par l'utilisation de données de registres géométriques et radiométriques. Nous avons exploré dans ce cas la qualité de l'association de l'agrosystème oasien à la physionomie exprimée. À Nefta, nous avons acquis une façon de décrypter, dans ces deux registres de données, l'information soutenue par le système référent. Leur capacité indicatrice du système est donc confirmée.

Un préalable essentiel à cette démarche est la connaissance des oasis acquise par les agronomes et la conceptualisation effectuée dans le cadre d'une approche systémique, ici de l'agrosystème oasien (Fargette et al., 2017b). La diversité de la relation agricole de l'homme au milieu a été ici capturée, analysée, décrite; la connaissance relative au concept d'agrosystème, construite; la typologie de systèmes de cultures, organisée. Le travail s'est nourri d'une compréhension synthétique des pratiques et savoir-faire, des objectifs et organisation de société, des initiatives libres ou contraintes, l'ensemble endossant le poids de l'histoire et de sa contingence. Les objets agricoles témoins et les signes observés mais aussi l'intangible sociétal sont pris en compte. Cet apport préalable pose le cadre systémique et apporte sens au signe. 


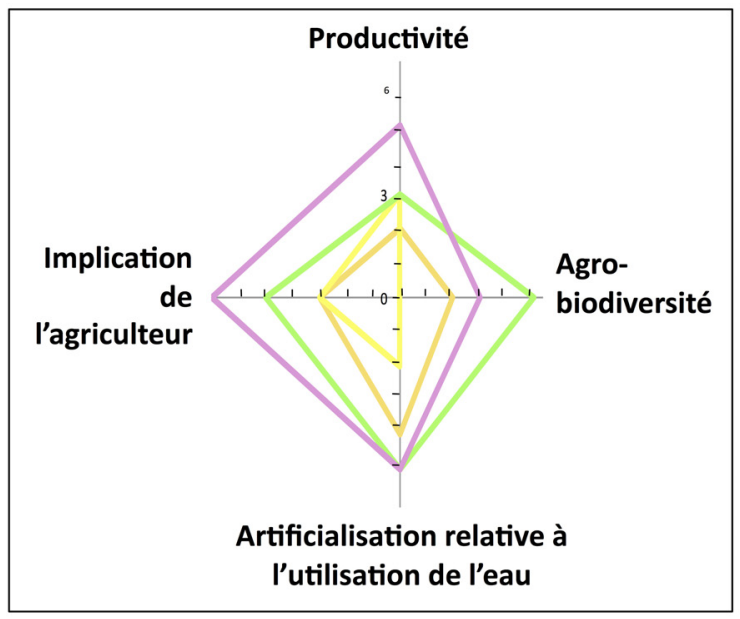

Code couleur: Types de système de culture

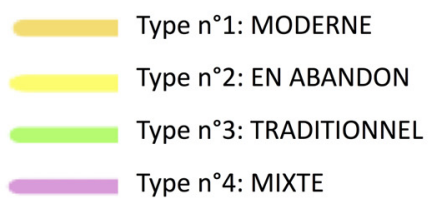

Echelle qualitative synthétique

0 : inexistant, 1 : très faible, 2 : faible, 3: assez faible, 4: moyen, 5 : assez élevé, 6 : élevé, 7: très élevé.

\section{Distribution des variables par thèmes}

Productivité : densité de palmiers, travail du sol, apport de fumier ; Agro-biodiversité : nombre de variétés de palmiers-dattiers, présence de fruitiers, présence de cultures basses (maraîchères et/ou fourragères) ; Artificialisation relative à l'utilisation de l'eau : volume d'eau d'irrigation, présence de bassin de collecte, de puits, de séguia ;

Implication de l'agriculteur : type de main d'œuvre mobilisée, temps de présence, renouvellement progressif des pieds de palmier, gestion des palmes sèches.

\section{Remarque: les choix de distribution se discutent (une même variable pouvant contribuer à plusieurs thèmes) ; l'intention ici n'est pas de caractériser chaque thème.}

Fig. 4. Représentation graphique du profil de chaque type de système de culture.

Fig. 4. Graphic representation of each cropping system type.

Tableau 1. Poids des variables géométriques et radiométriques du modèle de détection des types de système de culture. Table 1. Weight of the geometric and radiometric variables in the detection model of cropping system types.

\begin{tabular}{llllllll}
\hline & $\begin{array}{l}\text { Données } \\
\text { géométriques } \\
{[\mathrm{G}]}\end{array}$ & $\begin{array}{l}\text { Bandes } \\
\text { spectrales } \\
{[\mathrm{BS}]}\end{array}$ & $\begin{array}{l}\text { Indices } \\
\text { radiométriques } \\
{[\mathrm{IR}]}\end{array}$ & {$[\mathrm{G}]+[\mathrm{BS}]$} & {$[\mathrm{G}]+[\mathrm{IR}]$} & {$[\mathrm{BS}]+[\mathrm{IR}]$} & {$[\mathrm{G}]+[\mathrm{BS}]+[\mathrm{IR}]$} \\
\hline$\%$ de conformité & $53 \%$ & $60 \%$ & $60 \%$ & $64 \%$ & $65 \%$ & $60 \%$ & $69 \%$ \\
\hline
\end{tabular}

Le partage de connaissance et de savoir-faire, en interdisciplinarité (ici entre agronomie, socio-économie, géographie, géomatique et statistique), n'est possible qu'en montant en abstraction dans les raisonnements et les concepts, ce que nous ont ouvert le cadre de réflexion sur la systémique, l'agrosystème et son contenu sémantique d'une part, la sémiotique, «le sens des signes» et la relation systèmeempreinte d'autre part.

Plus largement, la relation théorique système-empreinte est généralisable à toute relation société-milieu. La démarche pratique qui s'intéresse à déceler l'empreinte du système dans la physionomie l'est aussi, tout autant que l'on dispose d'une compréhension systémique suffisante; il s'agit là d'un verrou important, relatif au manque possible de connaissance. On pourra, par exemple, à partir d'un registre d'observation choisi, suivre, pour une même plante, l'extension d'une épidémie affectant une culture, évaluer la production agricole d'une région, son degré de maturité et sa date de récolte, sa diversité variétale ou l'impact physiologique d'une sécheresse ( $c f$. Theia, 2017). Les apports disciplinaires assurent, dans chaque cas, la juste formalisation du système référent.

Les enjeux contemporains, tout particulièrement quand ils sont de nature interdisciplinaire (souveraineté alimentaire, activité économique et emploi d'une région), entrent de plainpied dans la problématique de suivi des oasis et de gestion des territoires en zones arides, qu'ils soient spécifiques à une problématique agricole (intensification, diversification, risque sanitaire) ou plus généraux, par exemple, questionnant le devenir des vocations oasiennes ou la qualité de vie de leurs sociétés (Fargette et al., 2017b) fortement soumises à des échanges diversifiés (économie de marché et touristique, urbanisation).

Les concepts, raisonnements et méthodes posés dans les Observatoires scientifiques en appui aux gestionnaires de territoire (OSAGE) par Loireau et al. (2017) sont compatibles avec la démarche présentée ici. Celle-ci a montré son potentiel dès ce stade encore exploratoire et le raisonnement à la base de la construction de la fonction d'association est indépendant du temps ou du lieu, donc applicable largement. Elle peut contribuer au suivi d'empreinte, via l'étude diachronique d'un continuum spatial de données, par exemple, issues d'images satellitaires. Elle est généralisable à toute question complexe de société. Un observatoire, saisi d'une question de société, peut $y$ avoir recours dans son processus scientifique d'observation et d'analyse, tout autant que soient respectées les conditions de référencement systémique et d'indépendance 
de données. La démarche proposée ici aurait sa place dans un observatoire OSAGE des territoires oasiens au plus près des sociétés (Fargette et al., 2017a).

Remerciements. Ce travail a bénéficié d'une aide de l'État français, gérée par l'Agence nationale de la recherche, au titre du programme «Investissements d'avenir » pour le projet EQUIPEX GEOSUD (ANR-10-EQPX-20). Le travail de terrain a bénéficié du support logistique et expert du Centre régional de recherche en agriculture oasienne (CRRAO) de Degache et du Commissariat régional du développement agricole (CRDA) de Tozeur.

\section{Références}

AGDOR. 2015. Sauver l'oasis de Nefta. https://nawaat.org/portail/ 2015/12/31/sauver-loasis-de-nefta/.

Barthes R. 1953. Les planches de l'encyclopédie. Le degré zéro de l'écriture. Nouveaux essais critiques. Paris: Le Seuil, pp. 89-105. Disponible sur http://ae-lib.org.ua/texts/barthes_nouveaux_essais_ critiques fr.htm $\# 2$.

Battesti V. 2005. Jardins au désert, évolution des pratiques et savoirs oasiens, Djérid tunisien. Paris: IRD éditions. Disponible sur https:// halshs.archives-ouvertes.fr/halshs-00004609v2.

Brunet R. 1974. Analyse des paysages et sémiologie. Éléments pour un débat. L'espace géographique 2: 120-126. Disponible sur http:// www.persee.fr/doc/spgeo_0046-2497_1974_num_3_2_1460.

Claval P. 1974. Géographie et sémiologie. L'espace géographique 2: 113-119. Disponible sur http://www.persee.fr/doc/spgeo_0046-2497_ 1974 num_3_2_1459?q=géographie\%20et\%20sémiologie.

Conforti J, Tonneau JP. 1999. Les systèmes de production oasiens- le cas des oasis du Djérid. In: Ferry M, Bedrani S, Greiner D, eds. Agroéconomie des oasis. Montpellier: CIRAD, pp. 103-115.

Deffontaines JP. 2004. L'objet dans l'espace agricole. Le regard d'un géoagronome. Natures Sciences Sociétés 12(3): 299-304. Disponible sur http://www.cairn.info/revue-natures-sciences-societes2004-3-page-299.htm.

Fargette M, Loireau M, Ben Kathra N, Kiari H, Libourel T. 2017a. Conceptual analysis of climate change in the light of societyenvironment relationships; observatories closer to both systems and societies. In: Serrao-Neumann S, Coudrain A, Coulter L, eds. Developing and communicating climate change information for decision making. Dordrecht, New York, Heidelberg, London: Springer.

Fargette M, Loireau M, Sghaier M, Raouani N, Libourel T. 2017b. The future of oases in Maghreb as through the prism of a systemic approach: which viability and coviability? In: Barrière $\mathrm{O}$, Morand S, Behnassi M, David G, Douzal V, Canete V, et al., eds. Coviability of social and ecological systems: reconnecting mankind to the biosphere in an era of global change. Dordrecht, New York, Heidelberg, London: Springer.

Kassah A. 2009. Oasis et aménagement en zones arides. Enjeux, défis et stratégies. In: Marlet S, Mekki I, eds. Gestion des ressources naturelles et développement durable des systèmes oasiens du Nefzaoua. Douz (Tunisie): Cirad. Disponible sur http://hal.cirad.fr/ cirad-00496143.

Loireau M, Fargette M, Desconnets JC, Khiari H. 2017. Observatoire scientifique en appui aux gestionnaires de territoire: entre abstraction OSAGE et réalité ROSELT/OSS. Cas ROSELT/OSS pour la lutte contre la désertification. Revue Internationale de Géomatique, Numéro spécial, Autour du concept d'observatoire en environnement, 30 p. Sous presse. DOI: 10.3166/rig.2017.00033.

Michelin, Y. 2008. L'approche sémiologique au service de la mise en évidence du lien produit agricole-paysage: l'exemple de l'AOC Saint-Nectaire. Disponible sur http://epublications.unilim.fr/ revues/as/3409.

Namsi A, Montarone M, Serra P, Ben Mahamoud O, Takrouni ML, Zouba A, et al. 2007. Manganese and brittle leaf disease of date palm tree. Journal of Plant Pathology 89(1): 125-136. Available from http://www.sipav.org/main/jpp/volumes/0107/010715.pdf.

Sébillotte M. 1990. Système de culture : un concept opératoire pour les agronomes. In Combe L, Picard D, eds. Les systèmes de culture. Paris: INRA, pp. 165-196. Disponible sur http://prodinra.inra.fr/ record/94106.

Theia. 2017. Pôle de données et de services surfaces continentales Theia. Agriculture. Disponible sur https://www.theia-land.fr/fr/ themes/agriculture.

Triki MA, Zoouba A, Khoualdia O, Ben Mahamoud O, Takrouni ML, Garnier M. 2003. Brittle leaf disease of date palms in Tunisia: biotic or abiotic disease? Journal of Plant Pathology 85(2): 71-79. Available from www.jstor.org/stable/41998127.

Citation de l'article : Fargette M, Loireau M, Raouani N, Kadri Zara M, Simon H, Libourel T, Sghaier M, Leibovici D. 2017. Modélisation de la diversité des systèmes de culture, de l'observation à la détection: cas de l'oasis de Nefta (Djérid, Tunisie). Cah. Agric. 26: 45009. 